\title{
Visible Light-Induced Transformation of Polymer Networks
}

\author{
Ali Bagheri ${ }^{\mathrm{a}, \mathrm{b}^{*}}$, Chris Bainbridge ${ }^{\mathrm{a}, \mathrm{b}}$ and Jianyong Jin ${ }^{\mathrm{a}, \mathrm{b}^{*}}$ \\ a School of Chemical Sciences, The University of Auckland, Auckland 1010, New Zealand \\ ${ }^{\mathrm{b}}$ Dodd-Walls Centre for Quantum and Photonic Technologies, Auckland 1010, New Zealand
}

Email: ali.bagheri@auckland.ac.nz

Email: j.jin@auckland.ac.nz 


\section{Experimental section}

\section{Materials}

Oligo(ethylene glycol) ether acrylate (OEGA) $\left(M_{\mathrm{n}}=480 \mathrm{gmol}^{-1}\right), 2$-hydroxyethyl acrylate (HEA), tetra (ethylene glycol) diacrylate (TEGDA), and bisphenol A ethoxylate diacrylate (Bis-EDA) were purchased from Sigma Aldrich and deinhibited by passing through a column of basic alumina. 5, 10, 15, 20-tetraphenyl-21H, 23H-porphine zinc (ZnTPP), N,N'methylenebisacrylamide, and S, S-Dibenzyl trithiocarbonate (DBTTC) were purchased from Sigma Aldrich and used as received. All the other reagents were used as received unless otherwise specified.

\section{Characterization}

All ${ }^{1} \mathrm{H}-\mathrm{NMR}$ spectra were recorded using a Bruker $400 \mathrm{MHz}$ spectrometer. All chemical shifts are reported in ppm $(\delta)$ relative to tetramethylsilane, referenced to the chemical shifts of residual solvent resonances.

Gel permeation chromatography (GPC) was performed with Waters GPC Breeze 2 system attached with Styragel HR 4 and HR 4E (300 mm $\times 7.8 \mathrm{~mm}$ each) Waters columns. THF was filtered through $0.22 \mu \mathrm{m}$ PTFE filter and used as eluent with the flow rate of $1.0 \mathrm{~mL} / \mathrm{min}$. The columns and RI detector were maintained at $30^{\circ} \mathrm{C}$. Polystyrene standards were used to plot a calibration curve.

Total monomer conversions of the prepared networks were measured based on the mass of the dry samples (after extensive washing and drying in the room temperature). The conversion of the newly incorporated OEGA monomer after photo-growth process was also measured based on the mass of the dry samples after extensive washing of networks in different solvents (such as DMSO, MeCN and tetrahydrofuran (THF)) for a minimum of $24 \mathrm{~h}$.

Swelling ratios of networks were measured in fully swollen state at room temperature: swelling ratio defined as $W_{w} / W_{d}$, where $W_{w}$ is the weight of a sample swollen in DMSO; $W_{d}$ is the weight of sample in the dry state. 


\section{EXPERIMENTAL SECTION}

General Procedures for Kinetics Studies of Photoredox-Catalyzed RAFT Polymerization Mediated by ZnTPP in the Presence of DBTTC

A typical kinetics procedure for RAFT polymerization of oligo(ethylene glycol) methyl ether acrylate) (OEGA) using [OEGA]:[DBTTC]:[ZnTPP] $=200: 1: 10^{-2}$ was set up as follows: a solution containing OEGA (1.65 g, $3.44 \mathrm{mmol}$ ), DBTTC (5.0 mg, $17.2 \mu \mathrm{mol})$, ZnTPP, (116.7 $\mu \mathrm{L}, 1.7 \times 10^{-4} \mathrm{mmol}$ ) from a $1 \mathrm{mg} / \mathrm{mL}$ stock solution, and dimethyl sulfoxide (DMSO) (1.7 $\mathrm{mL}, 2 \mathrm{M}$ concentration) was prepared. The reaction mixture divided into four different vials. The glass vials were covered in aluminum foil, sealed with a rubber septum and purged with nitrogen for $30 \mathrm{~min}$. The polymerization was carried under red LED light, while samples were taken at predetermined intervals, and analyzed by ${ }^{1} \mathrm{H}$ NMR and GPC (tetrahydrofuran (THF)) to determine the conversion, number-average molecular weight $\left(M_{\mathrm{n}, \mathrm{GPC}}\right)$ and molecular weight distribution $\left(M_{\mathrm{w}} / M_{\mathrm{n}}\right)$.

Photo-Expandable/Transformable Polymer Networks (PET-PNs) Formation via Visible Light-Induced Photoredox-Catalyzed Radical Polymerization, e.g. Network-4;

A typical experiment of a parent RAFT network formation was set up as follow: a glass vial was charged with OEGA (33.0 mg, $68.9 \mu \mathrm{mol})$, TEGDA (187.3 mg, $619.7 \mu \mathrm{mol})$, DBTTC (2.0 $\mathrm{mg}, 6.9 \mu \mathrm{mol})$, ZnTPP $\left(23.3 \mu \mathrm{L}, 3.44 \times 10^{-5} \mathrm{mmol}\right)$ from a $1 \mathrm{mg} / \mathrm{mL}$ stock solution, and DMSO (137.7 $\mu \mathrm{L}, 5 \mathrm{M}$ concentration). The glass vial was covered in aluminum foil, sealed with a rubber septum and purged with nitrogen for $20 \mathrm{~min}$. The polymerization was carried under a red LED light (12h). After polymerization the formed network was removed from the vial and washed in DMSO, THF and acetonitrile $(\mathrm{MeCN})$ to remove ZnTPP and any unreacted monomers. This process was also used to make networks with differing ratios of [TEGDA]: [OEGA] or using Bis-EDA as cross-linker. Note: The theoretical molecular weight (MW) of networks were assumed to be similar to the MW of polymer chain without crosslinking; and the target degree of polymerization for the photo-growth process calculated based on the theoretical $M_{\mathrm{n}}$ of the networks.

\section{Photo-growth of Parent PET-PN by Photoredox-Catalyzed Light Polymerization, e.g.} Network-4-I;

Photo-growth of a parent network (Network-4) was carried out as follow: a solution containing OEGA (660.9 mg, $1.37 \mathrm{mmol}), \operatorname{ZnTPP}\left(46.7 \mu \mathrm{L}, 6.88 \times 10^{-5} \mathrm{mmol}\right)$ from a $1 \mathrm{mg} / \mathrm{mL}$ stock solution, and DMSO $(275.4 \mu \mathrm{L}, 5 \mathrm{M}$ concentration) was prepared. The parent network was then soaked in this solution for 12 hours and degassed for 20 minutes at room temperature, 
before being irradiated under a red LED light (6h). After photo-growth reaction, the network was removed from the light source, and was extensively washed in DMSO, THF and MeCN to remove ZnTPP and unreacted monomers.

\section{General Procedures for Kinetics Studies of Photoiniferter-Mediated RAFT Polymerization of OEGA using DBTTC}

A typical kinetics procedure for RAFT polymerization of OEGA using [OEGA]: [DBTTC] $=$ 200: 1 was set up as follow: a solution containing OEGA (3.305 g, $6.885 \mathrm{mmol})$, DBTTC (10 $\mathrm{mg}, 34.4 \mu \mathrm{mol})$, and DMSO (1.3 mL, $5 \mathrm{M}$ concentration) was prepared. The reaction mixture divided into seven different vials. The glass vials were covered in aluminum foil, sealed with a rubber septum and purged with nitrogen for $30 \mathrm{~min}$. The polymerization was carried under blue LED light, while samples were taken at predetermined intervals, and analyzed by ${ }^{1} \mathrm{H}$ NMR and GPC (THF) to determine the conversion, number-average molecular weight $\left(M_{\mathrm{n}, \mathrm{GPC}}\right)$ and molecular weight distribution $\left(M_{\mathrm{w}} / M_{\mathrm{n}}\right)$.

\section{PET-PNs Formation via Photoiniferter-Mediated RAFT Polymerization; e.g. NT-13}

A typical experiment of a parent RAFT network was set up as follow: a glass vial was charged with OEGA (33.0 mg, $68.9 \mu \mathrm{mol})$, TEGDA (187.3 mg, $0.6197 \mathrm{mmol})$, DBTTC (2.0 mg, 6.9 $\mu \mathrm{mol})$, and DMSO (137.7 $\mu \mathrm{L}, 5 \mathrm{M}$ concentration). The glass vial was covered in aluminum foil, sealed with a rubber septum and purged with nitrogen for $20 \mathrm{~min}$. The polymerization was carried under a blue LED light (48 h). After polymerization the formed network was removed from the vial and washed in DMSO, THF and MeCN to remove ZnTPP and any unreacted monomers. Note: The theoretical molecular weight (MW) of networks were assumed to be similar to the MW of polymer chain without crosslinking; and the target degree of polymerization for the photo-growth process calculated based on the theoretical $M_{\mathrm{n}}$ of the networks.

\section{Photo-growth of Parent PET-PN using Photoiniferter-Mediated Polymerization; e.g. NT-}

\section{3-I}

Photo-growth of a parent network (NT-13; $\alpha=61 \%$ and initial mass $=47.6 \mathrm{mg}$ ) was carried out as follow: a solution containing OEGA $(230.6 \mathrm{mg}, 0.48 \mathrm{mmol})$ and DMSO $(96.4 \mu \mathrm{L}, 5 \mathrm{M}$ concentration) was prepared. The parent network was then soaked in this solution for 12 hours and degassed for 30 mins at room temperature, before being irradiated under a blue LED light (24 h). After photogrowth reaction, the network was removed from the light source, and was extensively washed in DMSO, THF and MeCN to remove ZnTPP and unreacted monomers. 


\section{Welding of Networks using Photo-RAFT Polymerization}

Welding of the RAFT networks was carried out as follows: two of the parent networks were swelled in a solution containing OEGA (1.32 g, $2.75 \mathrm{mmol}), N, N^{\prime}$-methylenebisacrylamide (2.12 mg, $0.013 \mathrm{mmol}), \mathrm{ZnTPP}\left(93.38 \mu \mathrm{L}, 1.37 \times 10^{-4} \mathrm{mmol}\right)$, and DMSO $(550 \mu \mathrm{L}, 5 \mathrm{M}$ concentration). The networks were then stacked one above the other, in a vial which was degassed for 20 minutes, before being irradiated under a red LED light for $12 \mathrm{~h}$. Similar experimental approach was employed for photoiniferter-mediated polymerization without addition of ZnTPP, while blue LED light was used as a light source. 


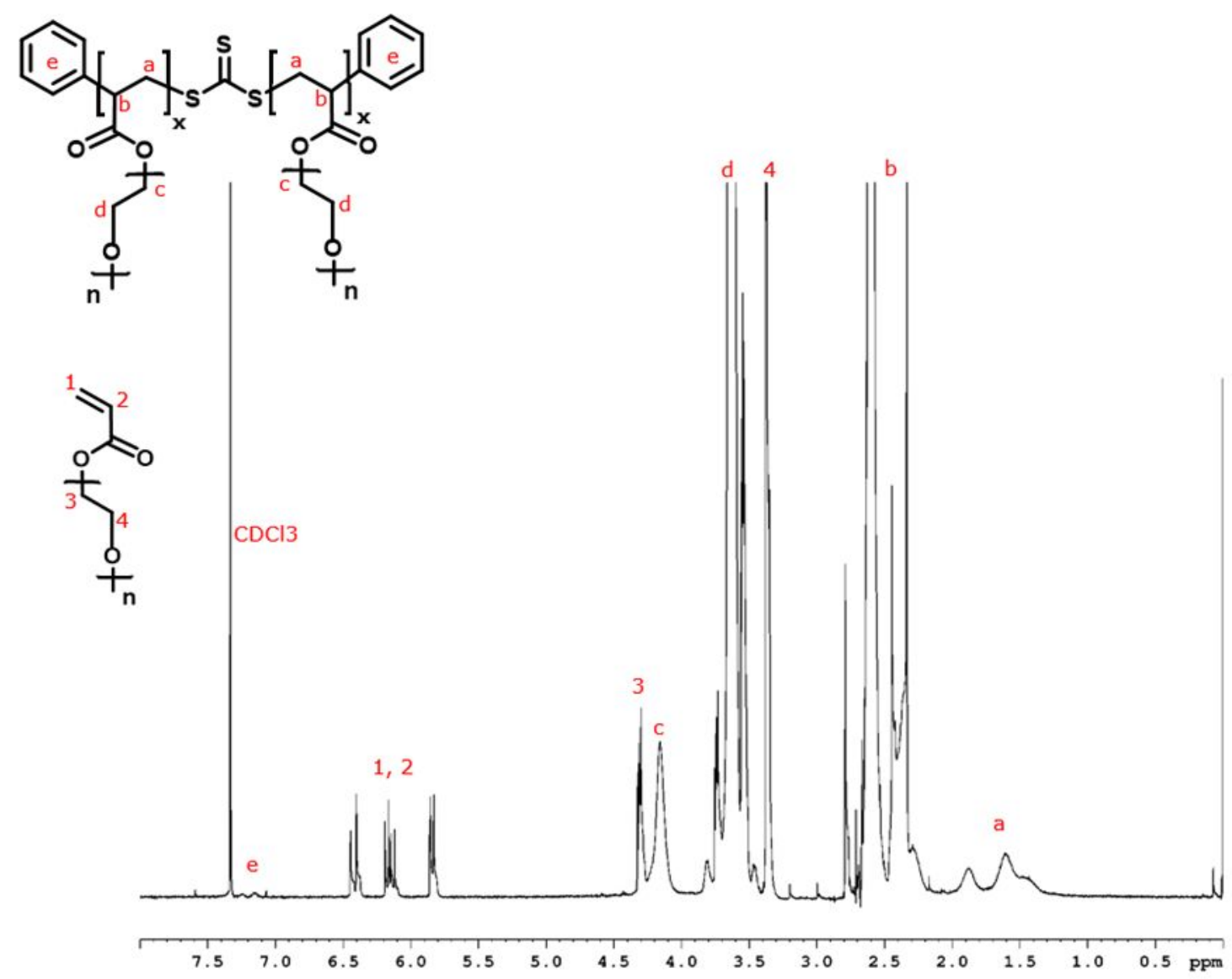

Figure S1. ${ }^{1} \mathrm{H}$ NMR analysis of POEGA produced by light induced $\left(\lambda{ }_{\max }=635 \mathrm{~nm}, 0.7\right.$ $\mathrm{mW} / \mathrm{cm}^{2}$ ) RAFT polymerization using a [OEGA]:[DBTTC]:[ZnTPP] $=200: 1: \mathbf{1 0}^{-\mathbf{2}}$ ratio. 


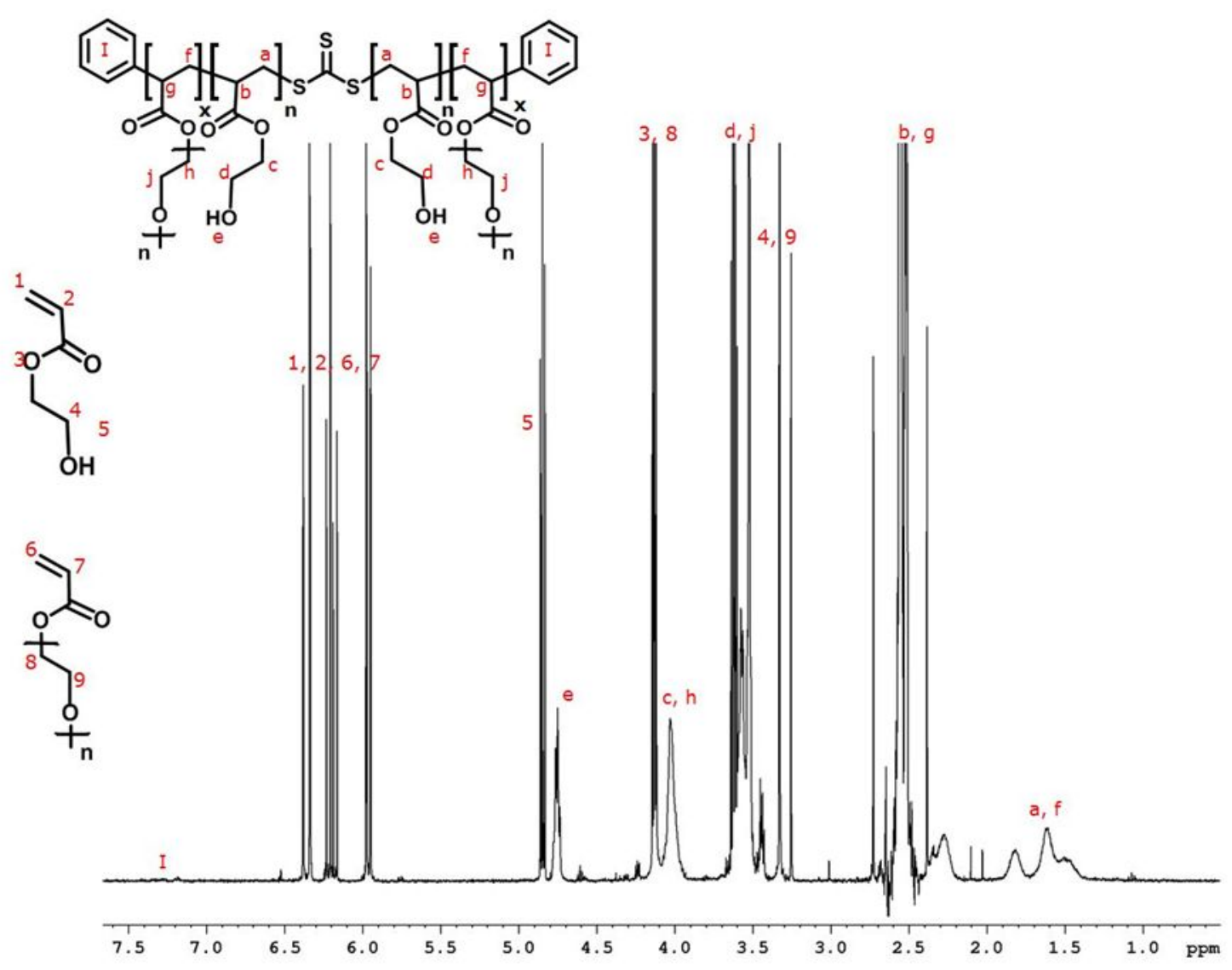

Figure S2. ${ }^{1} \mathrm{H}$ NMR analysis of macro-CTA chain extended with HEA produced by light induced (under red LED $\left(\lambda_{\max }=635 \mathrm{~nm}, 0.7 \mathrm{~mW} / \mathrm{cm}^{2}\right)$ ) RAFT polymerization using a [HEA]: [macro-CTA]: [ZnTPP] $=200: 1: 10^{-2}$. 


\section{Parent PET-PNs}

(a)

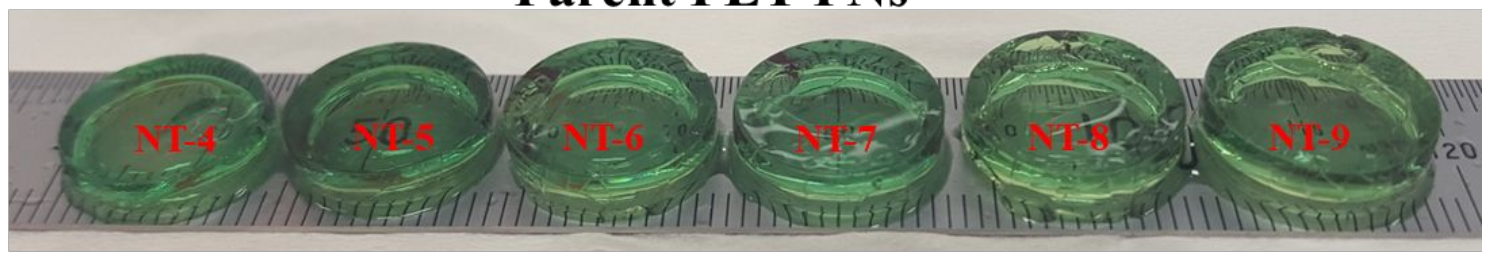

Daughter PET-PNs

(b)

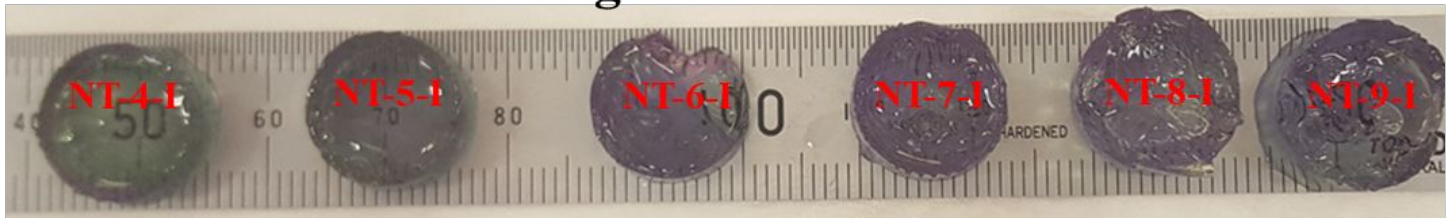

Figure S3. (a) Optical images of parent networks synthesized with different mole ratios of OEGA monomer and TEGDA cross-linking monomer under red light irradiation. (b) Optical images of daughter networks after photo-growth reaction ( $6 \mathrm{~h}$ under red light irradiation) using molar ratio of $[\mathrm{OEGA}]:\left[\right.$ network-TTC]:[ZnTPP] $=200: 1: 10^{-2}$. Note that images of daughter networks were taken after completion of reaction (before washing), showing higher amount of ZnTPP within the networks with higher swelling ratios. 
Table S1. Results of photoredox-catalyzed growth of networks, displayed by mass before and after growth with OEGA. ${ }^{a}$ Mass of gels in a dry state. ${ }^{b}$ Mass of gels in a fully swollen state in DMSO.

\begin{tabular}{lllll}
\hline Entry & $\begin{array}{l}\mathrm{a} \mathrm{W}_{\mathrm{D}}, \text { before growth } \\
(\mathrm{mg})\end{array}$ & $\begin{array}{l}{ }^{\mathrm{b}} \mathrm{W}_{\mathrm{W}}, \text { before growth } \\
(\mathrm{mg})\end{array}$ & $\begin{array}{l}{ }^{\mathrm{a}} \mathrm{W}_{\mathrm{D}}, \text { after growth } \\
(\mathrm{mg})\end{array}$ & $\begin{array}{l}{ }^{\mathrm{b}} \mathrm{W}_{\mathrm{W}}, \text { after growth } \\
(\mathrm{mg})\end{array}$ \\
\hline NT-4 & 156.8 & 300.5 & 276.3 & 1766.5 \\
NT-5 & 166.5 & 330.0 & 310.1 & 1854.6 \\
NT-6 & 155.1 & 320.0 & 331.0 & 1570.1 \\
NT-7 & 181.6 & 389.0 & 427.9 & 2012.4 \\
NT-8 & 170.1 & 388.7 & 365.9 & 1661.8 \\
NT-9 & 177.3 & 447.4 & 406.5 & 1732.6 \\
\hline
\end{tabular}




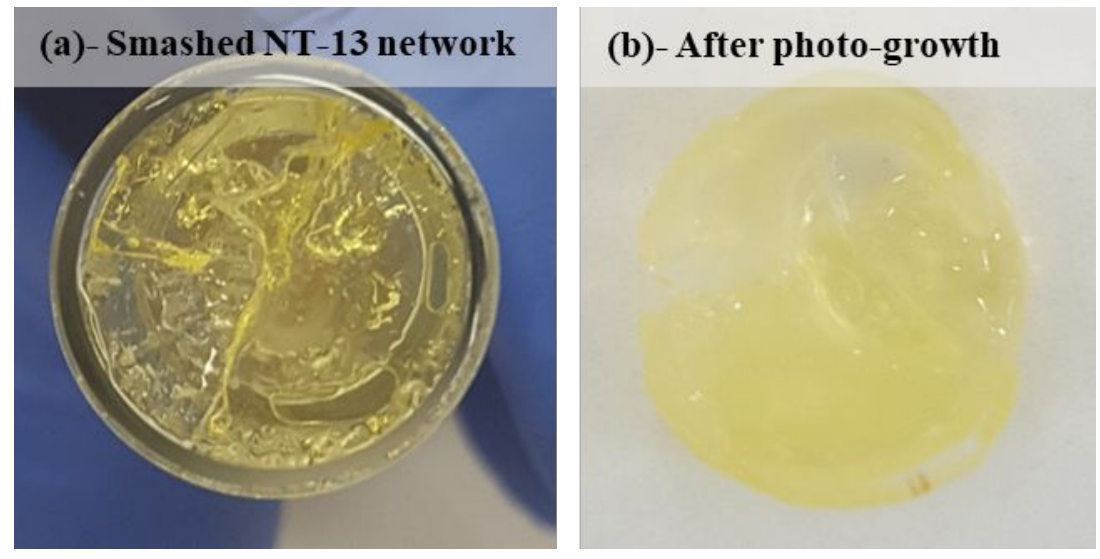

Figure S4. Applying visible light-induced photoiniferter-mediated polymerization for network welding: optical image of (a) completely smashed crosslinked network (NT-13) combined into (b) one piece after exposure to blue LED light in the absence of catalyst. 